Methods Systematic methods were used to identify relevant studies, assess study eligibility for inclusion and evaluate study quality. Cohort studies of adults with a primary diagnosis of lung cancer, published in peer-reviewed English language journals up to 2011, were examined. All studies reporting rates of receipt of any treatment for lung cancer according to a measure of SES were included in the review. Studies that reported odds ratios for receipt of treatment, adjusted for at least age and sex, were included in the metaanalysis. Subgroup analyses by healthcare system (universal healthcare system or insurance-based system), histology and stage were conducted.

Results From the initial 1345 studies identified, 46 studies were included in the review and 29 in the meta-analysis.

Socio-economic inequalities in receipt of lung cancer treatment were observed. Low SES was associated with a reduced likelihood of receiving any treatment $(\mathrm{OR}=0.79$, $\mathrm{CI}(0.74$ to 0.84$) \mathrm{p}<0.001)$, surgery $(\mathrm{OR}=0.71$ (CI 0.65 to 0.77$), \mathrm{p}<0.001)$ and chemotherapy $(\mathrm{OR}=0.81$ (CI 0.73 to 0.91 ), $\mathrm{p}<0.001$ ), but not radiotherapy $(\mathrm{OR}=0.95$ (CI 0.84 to 1.07$), \mathrm{p}=0.41)$, for lung cancer. The association was found in both insurance-based and universal healthcare systems and remained when stage and histology were taken into account for receipt of surgery.

Conclusion This systematic review and meta-analysis found that lung cancer patients living in more socio-economically deprived circumstances were less likely to receive any type of treatment, surgery and chemotherapy. These inequalities cannot be accounted for by socio-economic differences in stage at presentation or by type of healthcare system. Further investigation is required into the patient, clinician and system factors that may contribute to socio-economic inequalities in receipt of lung cancer care and how these inequalities may impact on survival, and also into how to reduce such inequalities.

\section{Poster Programme}

\section{PS01 ASSOCIATIONS OF HEALTH, PHYSICAL ACTIVITY AND WEIGHT STATUS WITH MOTORISED TRAVEL AND TRANSPORT CARBON DIOXIDE EMISSIONS}

doi:10.1136/jech-2012-201753.100

${ }^{1,2} \mathrm{~A}$ Goodman, ${ }^{3} \mathrm{C}$ Brand, ${ }^{2,4} \mathrm{D}$ Ogilvie. ${ }^{1}$ Nutrition and Public Health Interventions Research Department, LSHTM, London, UK; ${ }^{2}$ UKCRC Centre for Diet and Activity Research (CEDAR), Cambridge, UK, ${ }^{3}$ Environmental Change Institute and Transport Studies Unit, University of Oxford, Oxford, UK; ${ }^{4}$ MRC Epidemiology Unit, Cambridge, UK

Background Motorised travel and associated carbon dioxide $\left(\mathrm{CO}_{2}\right)$ emissions generate substantial health costs, many of which disproportionately affect socio-economically disadvantaged groups. These health costs may include contributing to rising obesity levels. Obesity has in turn been hypothesised to increase motorised travel and/ or $\mathrm{CO}_{2}$ emissions, both because heavier people may use motorised travel more and because heavier people may choose larger and less fuel-efficient cars. These hypothesised associations have not been examined empirically, however, nor has previous research examined associations with other health characteristics. Recent years have, however, seen increasing research and policy attention to the potential 'co-benefits' of pursuing policies which simultaneously enhance public health and promote environmental sustainability. We therefore aimed to examine how and why weight status, health, and physical activity are associated with transport $\mathrm{CO}_{2}$ emissions.

Methods 3463 adults (18-91 years, 45\% male) completed questionnaires in the baseline iConnect survey at three study sites in the UK, self-reporting their health, weight, height and past-week physical activity. Seven-day recall instruments were used to assess travel behaviour and, together with data on car characteristics, were used to estimate $\mathrm{CO}_{2}$ emissions. We used path analysis to examine how far active travel, motor travel and car engine size mediated associations between health characteristics and $\mathrm{CO}_{2}$ emissions.

Results $\mathrm{CO}_{2}$ emissions were higher in overweight or obese participants (multivariable standardized probit coefficients $0.16,95 \% \mathrm{CI}$ $0.08,0.24$ for overweight vs. normal; $0.16,95 \%$ CI $0.04,0.28$ for obese vs. normal). Lower active travel and, particularly for obesity, larger car engine size explained $19-31 \%$ of this effect, but most of the effect was directly mediated by greater motorised travel distance. Walking for recreation and leisure-time physical activity predicted higher motorised travel distance and therefore higher $\mathrm{CO}_{2}$ emissions, while active travel predicted lower $\mathrm{CO}_{2}$ emissions. Poor health and illness did not independently predict $\mathrm{CO}_{2}$ emissions.

Conclusion Establishing the direction of causality between weight status and travel behaviour requires longitudinal data, but the engine size association suggests at least a potential causal effect of obesity on $\mathrm{CO}_{2}$ emissions. More generally, transport $\mathrm{CO}_{2}$ emissions are differently associated with different health characteristics, including associations between a health good and an environmental harm (recreational physical activity and high emissions). Thus health-environmental 'co-benefits' cannot be assumed. Instead, attention should also be paid to identifying and mitigating potential areas of tension, for example promoting low-carbon recreational activity.

\section{PSO2 "WE CAN ALL JUST GET ON A BUS AND GO": RETHINKING INDEPENDENT MOBILITY IN THE CONTEXT OF THE UNIVERSAL PROVISION OF FREE BUS TRAVEL TO YOUNG LONDONERS}

doi:10.1136/jech-2012-201753.101

${ }^{1} \mathrm{~A}$ Goodman, ${ }^{2} \mathrm{~A}$ Jones, ${ }^{3} \mathrm{H}$ Roberts, ${ }^{4 R}$ Steinbach, ${ }^{2} \mathrm{~J}$ Green. ${ }^{1}$ Department of Nutrition and Public Health Intervention Research, LSHTM, London, UK; ${ }^{2}$ Department of Health Services Research Policy, LSHTM, London, UK; ${ }^{3}$ General and Adolescent Paediatrics Unit, UCL Institute of Child Health, London, UK; ${ }^{4}$ Departmental of Social Environmental Health Research, LSHTM, London, UK

Background Recent years have seen increasing attention to 'independent mobility' as a determinant of children's physical health and psychosocial development. Previous research, however, largely frames independent mobility as a matter of having parental permission to travel without adults. It also predominantly focuses upon walking any cycling trips in the local area by young children. We therefore aimed to extend the independent mobility literature by examining mobility on public transport, mobility beyond the local area and mobility by adolescents. For this we use as a case study the recent provision of universal free bus travel to all young people in London, UK. We argue that idea of independent mobility can usefully be situated within the broader conception of opportunity and process freedoms which underpin Amartya Sen's influential 'capabilities approach' to human development.

Methods As part of the On the buses study, 118 young Londoners (age 12-18, 65 females) took part in 43 in-depth interviews (group size 1-3, 61 individuals) and 10 focus groups (group size 4-8, 57 individuals). Interviews and focus groups elucidated tacit, or everyday, influences on and effects of young people's transport mode choices. We analysed this data qualitatively, drawing on techniques from the constant comparative method, including initial microlevel open coding and an iterative approach to identifying and refining emerging conceptual categories.

Results Free bus travel enhanced young Londoners' capability to shape their daily mobility, both directly by increasing financial access and indirectly by facilitating the acquisition of the necessary skills, travelling companions and confidence. These capabilities in turn extended both opportunity freedoms (e.g. facilitating non"necessary" recreational and social trips) and process freedoms (e.g. feeling more independent by decreasing reliance on parents). Moreover, the universal nature of the entitlement often seemed crucial as 
it meant that free bus travel was typically held by all of a peer group. This rendered buses a socially inclusive way for groups to travel and spend time together, thereby enhancing group-level capabilities.

Conclusion We believe this attention to individual and group-level capabilities for self-determination provides the basis for a broader and more child-centered view of independent mobility than is typi$\mathrm{cal}$ in health research. The importance of the universal nature of the entitlement to free bus travel also provides an example of how policy interventions with universal coverage may have effects which are more than the 'sum of the parts' of alternative, targeted approaches.

\section{PSO3 SYSTEMATIC REVIEW AND META-ANALYSIS OF SCHOOL- BASED INTERVENTIONS TO REDUCE BODY MASS INDEX}

doi:10.1136/jech-2012-201753.102

HV Lavelle, JP Pell, DF Mackay. Centre for Population Health Sciences, University of Glasgow, Glasgow, UK

Background Childhood obesity predisposes to adult obesity and increases the risk of many diseases. Schools provide a vehicle to deliver public health interventions to all children.

Methods Medline and Embase were used to undertake a systematic review of published studies on school-based interventions aimed at reducing body mass index (BMI) of children $\leq 18$ years. Preferred reporting items for systematic review and meta-analyses guidelines were followed, and eligible studies subjected to a random effects meta-analysis.

Results Between 1991 and 2010, 43 published studies provided 60 measurements of effect. The pooled effect was a 0.17 (95\% CI: 0.08 , $0.26, P<0.001)$ reduction in BMI. Heterogeneity was high $\left(I^{2}=93.4 \%\right)$ but there was no significant small study bias (Egger's test, $P=0.422$ ) nor significant variation by length of follow-iup. The intervention comprised physical activity only in $11(26 \%)$ studies, education only in three (7\%), and combinations of these and improved nutrition in the remaining 29 (67\%). On stratified analysis, physical activity used in isolation $(-0.13,95 \% \mathrm{CI}:-0.22,-0.04, P=0.001)$ or combined with improved nutrition $(-0.17,95 \%$ CI: $-0.29,-0.06, P<0.001)$ was associated with significant improvements in BMI. Interventions targeted at overweight/obese children reduced their BMI by 0.35 ( $95 \%$ CI: $0.12,0.58, P=0.003$ ). Those delivered to all children reduced it by 0.16 (95\% CI: $0.06,0.25, P=0.002)$.

Conclusion There is growing evidence that school-based interventions that contain a physical activity component may be effective in helping to reduce BMI in children.

\section{PSO4 VALIDITY OF CANCER DIAGNOSIS IN A PRIMARY CARE DATABASE COMPARED WITH LINKED CANCER REGISTRATIONS IN ENGLAND. POPULATION-BASED COHORT STUDY}

doi:10.1136/jech-2012-201753.103

${ }^{1} \mathrm{~A}$ Dregan, ${ }^{2 T}$ Murray-Thomas, ${ }^{3} \mathrm{H}$ Moller, ${ }^{1} \mathrm{M}$ Gulliford. ${ }^{1}$ Primary Care and Public Health Sciences, King's College London, London, UK; ${ }^{2}$ General Practice Research Database Group, MHRA, London, UK; ${ }^{3}$ Thames Cancer Registry, King's College London, London, $U K$

Background Electronic health records from primary care are increasingly utilised as a resource for epidemiological research in cancer. The present study aimed to evaluate the validity of cancer diagnoses recorded in a primary care database compared with cancer registry data in England.

Methods Eligible cancer codes were reviewed and agreed by two epidemiologists with experience in cancer and primary care. The eligible cohort comprised 42,556 participants, registered with English general practices in the General Practice Research Database
(GPRD) that consented to cancer registry linkage, who presented with haematuria, haemoptysis, dysphagia or rectal bleeding or were diagnosed with cancer of the lung, urinary tract, oesophagus/stomach, or colon/rectum between 2002 and 2006. Cancer registry (CR) records were linked through a unique identifier by a third party. Cancer registry and primary care records were compared for cancer diagnosis, date of cancer diagnosis and death.

Results There were 5,429 cancer diagnoses in GPRD and 5,710 in the CR, with 5,216 (91\% of CR total) diagnosed in both sources. There were 494 (9\%) diagnosed in CR but not in GPRD and 213 (4\%) that were diagnosed in GPRD but not CR. The predictive value of a GPRD cancer diagnosis was $96 \%$ for lung cancer, $92 \%$ for urinary tract cancer, $96 \%$ for gastro-oesophageal cancer and $98 \%$ for colorectal cancer. 'False negative' primary care records were sometimes accounted for by registration end dates being shortly before cancer diagnosis dates. The median (interquartile range) difference in date of cancer diagnosis (CR minus GPRD) was -11 (-30 to 6) days. Death records were consistent for the two sources for 3,337/3,397 (99\%) of cases.

Conclusion Recording of cancer diagnosis and mortality in primary care electronic records is generally consistent with cancer registrations in England. Linkage studies must pay careful attention to selection of codes to define eligibility and timing of diagnoses in relation to beginning and end of record.

\section{PSO5 MEN WITH PROSTATE CANCER MAKE POSITIVE DIETARY CHANGES FOLLOWING TREATMENT IN A RANDOMISED TRIAL: A PROSPECTIVE COHORT STUDY}

doi:10.1136/jech-2012-201753.104

${ }^{1} \mathrm{KNL}$ Avery, ' $\mathrm{JL}$ Donovan, 'R Gilbert, 'M Davis, 'P Emmett, 'E Down, ${ }^{2} \mathrm{~S}$ Oliver, ${ }^{3} \mathrm{DE}$ Neal, ${ }^{4} \mathrm{FC}$ Hamdy, ${ }^{\mathrm{J} A}$ Lane. ' $S$ School of Social and Community Medicine, University of Bristol, Bristol, UK; ${ }^{2}$ Department of Health Sciences, University of York, York, UK; ${ }^{3} \mathrm{Oncol}$ ogy Centre, Addenbrooke's Hospital, Cambridge, UK; ${ }^{4}$ Department of Surgical Sciences, University of Oxford, Oxford, UK

Background Prostate cancer (PC) is the second most frequently diagnosed cancer of men worldwide. A healthy diet may improve clinical outcomes but there is currently no disease-specific dietary advice available to aid survivorship after a PC diagnosis. The effect of a PC diagnosis on men's diet remains uncertain because, although cancer survivors report dietary changes, the direction and magnitude of changes are poorly understood. This study examined dietary changes in men before and after treatment for PC within the Prostate Testing for Cancer and Treatment (ProtecT) randomised trial.

Methods This was a prospective cohort study embedded within the Protec T randomised trial of treatments for PC. Participants were men aged 50-69 years tested for PC in primary care centres in nine areas of the UK. 3935 men completed a validated food frequency questionnaire before diagnosis and 678 with localised disease repeated the questionnaire one year later (response $82.7 \%$ ) Pre-diagnosis dietary intakes of men with different diagnoses subsequently (negative diagnosis, at risk, localised PC, advanced PC) were compared using linear regression. Dietary changes after a diagnosis of localised PC were examined using non-parametric tests. Effects of cancer treatments (radical prostatectomy, conformal external beam radiotherapy or active monitoring) were examined using linear regression.

Results Before diagnosis, all men consumed largely similar amounts of key nutrients and foods. The diagnosis of localised PC led to dietary changes in $29.2 \%$ of men. Changes observed were generally in a healthy direction, with 234 (34.7\%) men eating more fresh tomatoes $(\mathrm{p}<0.0001)$ and $156(23.5 \%)$ more tomato products $(p=0.01) .271(40.0 \%)$ men consumed more protein $(p<0.0001)$ and $193(28.6 \%)$ more fruit/vegetable juice $(p<0.0001)$. Less macronutrients were obtained from dairy products $(p<0.01)$. Men undergoing 